\title{
Could inter-set stretching increase acute neuromuscular and metabolic responses during resistance exercise?
}

\author{
Ubiratan Contreira Padilha (1), Amilton Vieira (1), Denis Cesar Leite Vieira (1,2), \\ Filipe Dinato De Lima (1), Valdinar Araújo Rocha Junior (3,4), James J Tufano (5), \\ Martim Bottaro (1)
}

(1) Physical Education College, University of Brasilia, DF, Brazil; (2) Department of Physical Education, UDF - University, Brasilia, DF, Brazil; (3) National Police Academy, Brasilia, DF, Brazil; (4) Department of Electrical Engineering, University of Brasilia, DF, Brazil; (5) Faculty of Physical Education and Sport, Charles University, Prague, Czech Republic

This article is distributed under the terms of the Creative Commons Attribution Noncommercial License (CC BY-NC 4.0) which permits any noncommercial use, distribution, and reproduction in any medium, provided the original author(s) and source are credited.

\begin{abstract}
This study investigated the acute effects of inter-set static stretching (ISS) during resistance exercise (RE) on the subsequent neuromuscular and metabolic responses. Twelve resistancetrained men performed three different knee extension RE protocols comprised of seven sets of 10 repetitions in a counterbalanced fashion. The three protocols were: 1) ISS (subjects performed $25 \mathrm{sec}$ of quadriceps stretching between sets during $40 \mathrm{sec}$ rest interval); 2) control (CON, subject passively rested between sets for $40 \mathrm{sec}$ ); 3) traditional (TRA, subject passively rested between sets for $120 \mathrm{sec})$. Total work was lower $(\mathrm{p}<0.05)$ in ISS than CON and TRA $(\mathrm{p}<0.05)$. The fatigue index was greater $(\mathrm{p}<0.05)$ in ISS compared with CON and TRA. ISS also resulted in lower ( $<$ 0.05) electromyography (EMG) amplitude during the 6th and 7th sets compared with TRA. Additionally, EMG frequency was lower $(\mathrm{p}<0.05)$ from the 3rd to 5th sets during ISS compared to CON, and from the 3rd to 7th sets compared to TRA. Muscle swelling and blood lactate similarly increased $(\mathrm{p}>0.05$ ) in response to all protocols. These results indicate that ISS negatively impacts neuromuscular performance, and does not increase the metabolic stress compared to passive rest intervals.
\end{abstract}

Key Words: Inter-set static stretching, strength exercise, electromyography, training volume, muscle swelling.

Eur J Transl Myol 29 (4): 293-301, 2019

Muscle hypertrophy and strength gains are the most desirable adaptations by resistance-trained individuals. Thus, a variety of strength-training (ST) methods and strategies have been developed to optimize these adaptations. $^{1-3}$ For example, with the purpose of stimulating mechanical, hormonal, and/or metabolic responses, advance resistance-trained individuals have been performing intra-set static stretching (ISS). ${ }^{4-6}$ Although the practice of ISS is common, there are few studies that investigated the chronic effects of ISS on muscle strength and hypertrophy, and the results are controversial. $^{4,7}$ For example, Evangelista et al. ${ }^{7}$ demonstrated that ISS was more effective to increase vastus lateralis muscle size than training using passive rest interval (TRA). However, the increases in rectus femoris, biceps, and triceps muscle sizes were similar between ISS and TRA. In addition, TRA appears as effective as ISS for improving muscle strength. ${ }^{4,7}$ Thus, like many other resistance training methods, there is no clear evidence that ISS is more effective than TRA with regard to hypertrophic and strength adaptations. ${ }^{4,7}$ Thus, this disparity between resistance training practice and experimental results has sparked a debate regarding the use of ISS to elicit strength gains and hypertrophy with resistance exercise. In spite of doubts about the efficiency of ISS for optimizing ST adaptations, the recommendations for performing ISS between sets is predicated on some physiological effects on skeletal muscle. ${ }^{4,5}$ Among the many physiologic changes are the increase of metabolites formation, accumulation of lactate, inorganic phosphate and hydrogen ions, and acute muscle hypoxia. ${ }^{8,9}$ These effects may potentiate the release of anabolic hormones and enhances anabolic signaling. ${ }^{8-10}$ Additionally, metabolites accumulation favors muscle swelling, ${ }^{8,11}$ that increases the pressure against the cytoskeleton and cellular membrane, 
disrupting cellular integrity and promoting anabolic intracellular signaling for muscle repair. ${ }^{8,11,12}$ Because stretch disrupts regional blood flow and reduces oxygen delivery to the muscle, it can favor metabolite formation and accumulation; thus, in theory, performing ISS might result in lactate accumulation and acute muscle swelling. ${ }^{5,11,13,14}$ However, byproduct accumulation and acute muscle swelling are not the only factors contributing to hypertrophy and strength adaptations. ${ }^{8,9,12}$ In fact, strength and power adaptation seems also to be mediated by mechanical stimuli and their interaction with other hormonal and metabolic factors. In this sense, the recruitment of higher number of motor units and an increase in firing frequency are also important factors in muscle growth and strength gains., ${ }^{9,15}$ According to previous reports, to perform stretching before maximal contraction reduces amplitude of electromyography (EMG), frequency and force production. ${ }^{16-18}$ Fowles et al. ${ }^{16}$ reported a decrease of $28 \%$ in the maximal voluntary contraction (MVC) of the plantar flexors and $15 \%$ of the EMG amplitude during MVC after a stretching protocol. Furthermore, Kay et al. ${ }^{18}$ showed a reduction in peak flexor moment after stretch, as well as a good correlation between the decrease in EMG amplitude and peak flexor moment. In contrast, Trajano et al. ${ }^{17}$ reported an increase in EMG amplitude during submaximal sustained isometric contractions after stretch, but the increase in EMG amplitude was accompanied by the decrease in EMG median frequency. Thus, it appears that stretch negatively influence mechanical stimuli during ST. However, studies reported that implementing ISS may increase the neuromechanical and metabolic stimuli by adding to total session time under tension to the session..$^{4,5}$ It is important to note, that the aforementioned influence of stretching on acute neuromuscular adaptations were based on experimental studies with stretch performed before only one maximal muscle action with short duration, ${ }^{16,18}$ or sustained submaximal isometric contractions, ${ }^{17}$ that are different of repeated dynamic muscle contractions generally applied during ST. Therefore, considering that the results of chronic studies on ISS are controversial, and the acute studies on the effects of stretching on neuromuscular performance do not resemble application in ST routines, ${ }^{19,20}$ it remains unclear whether the ISS applied to ST routines will be able to influence the physiologic changes associated with neuromuscular adaptations. In addition, it is necessary to analyze if the increase in time under tension by stretching will compensate for the decrement in mechanical stimuli such as force and work output. Thus, the purpose of this study was to determine the acute effects of inter-set agonist static stretching on mechanical stimuli (i.e, force production, work output, EMG amplitude, EMG median frequency, and muscle fatigue) and metabolic factors (i.e, muscle swelling, and lactate) in resistance-trained men.

\section{Materials and Methods}

\section{Participants}

Twelve resistance-trained men $(29.0 \pm 6.1$ years; $84.4 \pm$ $10.3 \mathrm{~kg} ; 1.78 \pm 0.06 \mathrm{~m})$ with at least one year of ST experience $(5.4 \pm 5.1$ years of experience; $4.0 \pm 0.1$ day'week ${ }^{-1}$ ) completed all experimental sessions and were included in the analysis. To be included in this study, participants must have been free from neuromuscular, metabolic, hormonal or cardiovascular problems. In addition, they should not be taking any medication that influences hormonal and neuromuscular function. They were instructed to not engage in any vigorous physical activity or unaccustomed exercise and to not take medications or food supplements during the entire study period. All procedures were approved by the University of Brasilia Institutional Review Board (2.103.261/2017) and were performed in accordance with

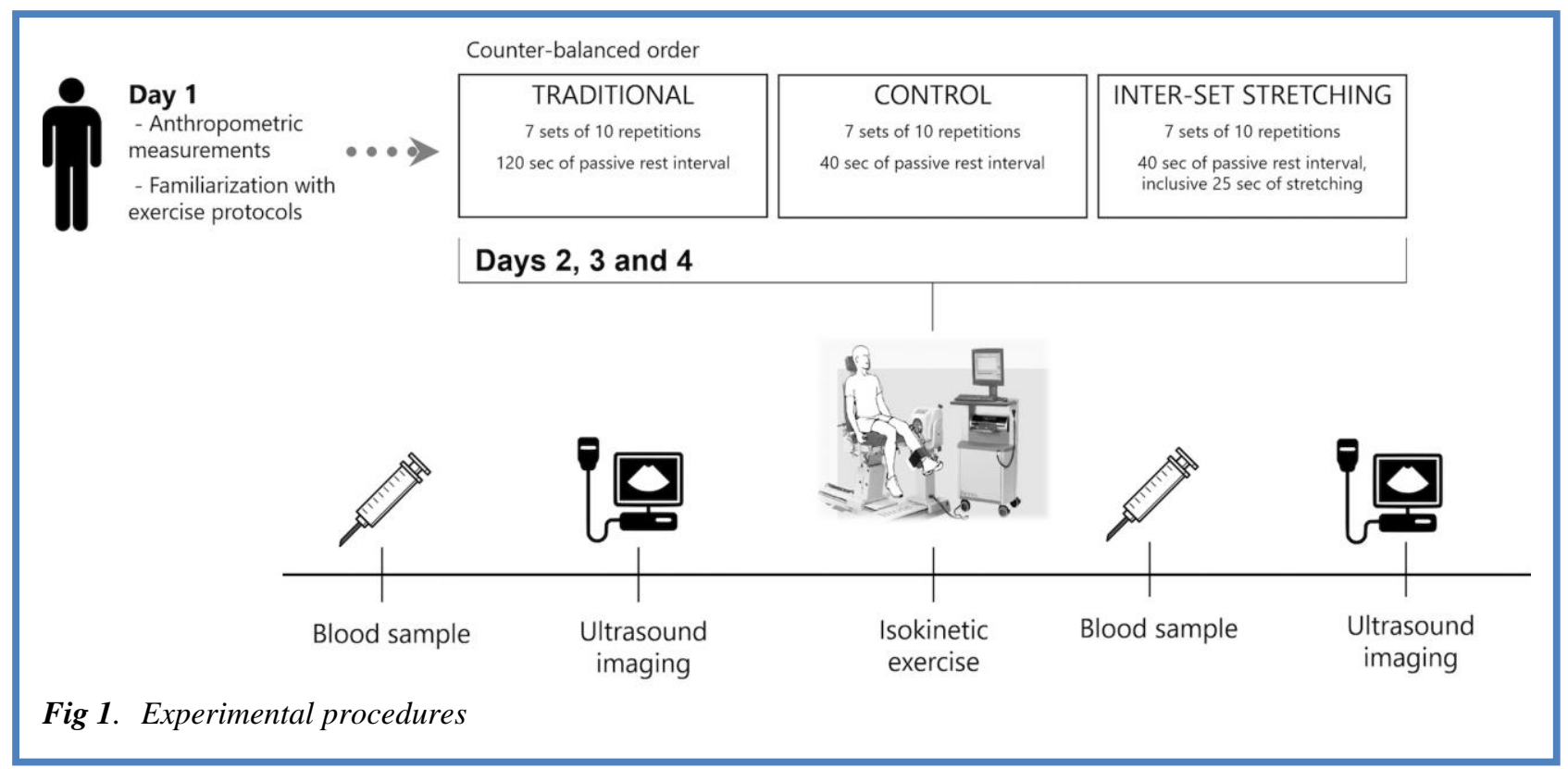


the Declaration of Helsinki. Informed consent was obtained from all individuals included in the study.

\section{Experimental procedures}

The experimental procedures are depicted in Figure 1. Participants reported to the laboratory on four occasions (from 72 to $196 \mathrm{~h}$ apart). During the first visit, anthropometric measurements and familiarization procedures were performed (four sets of 10-isokinetic knee extensions at $90^{\circ} \cdot \mathrm{s}^{-1}$ with 60 seconds of rest interval, static stretching was performed between $2^{\text {nd }}$ and $3^{\text {rd }}$ sets). In the following three visits, the three ST protocols (seven sets of 10 isokinetic knee extensions) were completed in counter-balanced order: 1) ISS (subjects performed $25 \mathrm{sec}$ of quadriceps stretching between sets during the $40 \mathrm{sec}$ rest interval); 2) control (CON, subject passively rested between sets for $40 \mathrm{sec}$ ); 3) traditional (TRA, subject passively rested between sets for $120 \mathrm{sec}$ ). Total work, peak torque, fatigue index, EMG activity, muscle swelling, and blood lactate responses were compared between the three protocols. The exercise protocol of isokinetic resistance exercise was chosen because the dynamometer allows a precise and reliable measure of force production and work output in each contraction throughout the protocol.

\section{Exercise Protocols}

To avoid circadian influences, participants performed the three exercise protocols at the same time of day $( \pm 1$ hour). All protocols were performed on an isokinetic dynamometer in a counter-balanced order (System 4, Biodex Medical, Inc., Shirley NY, USA). The lever arm was fixed $3 \mathrm{~cm}$ above the lateral malleolus, and gravity correction was performed according to the manufacturer's procedures. As a warm-up, participants were allowed to perform three isometric knee extensions progressively increasing effort from 50 to $100 \%$ of their maximal perceived effort. To specifically investigate the effects of ISS, all exercise protocols included seven sets of 10 isokinetic knee extensions at $60^{\circ} \cdot \mathrm{s}^{-1}$. Participants were verbally encouraged to exert maximal effort during all repetitions. The range of motion was set from $5^{\circ}$ to $90^{\circ}$ of knee flexion $\left(0^{\circ}=\right.$ full extension $)$. During the $40 \mathrm{sec}$ inter-set rest intervals of the ISS protocol, participants were removed from the dynamometer and performed static stretching of the agonist knee extensor muscles. The static stretching was done with the knee supported on a padded table by holding in stretch position at a standardized stretch intensity of slight discomfort (Figure 2). ${ }^{21}$ The time course of $40 \mathrm{sec}$ ISS rest interval protocol was composed of $5 \mathrm{sec}$ for the volunteer to move from the dynamometer to the padded table, $25 \mathrm{sec}$ of ISS, and $10 \mathrm{sec}$ to move the subject back to the dynamometer. This process was repeated for the remainder of the protocol (i.e. six sets of inter-set stretching were done). During the CON and TRA protocols, individuals passively rested for 40 or $120 \mathrm{sec}$, respectively. After the last set of knee

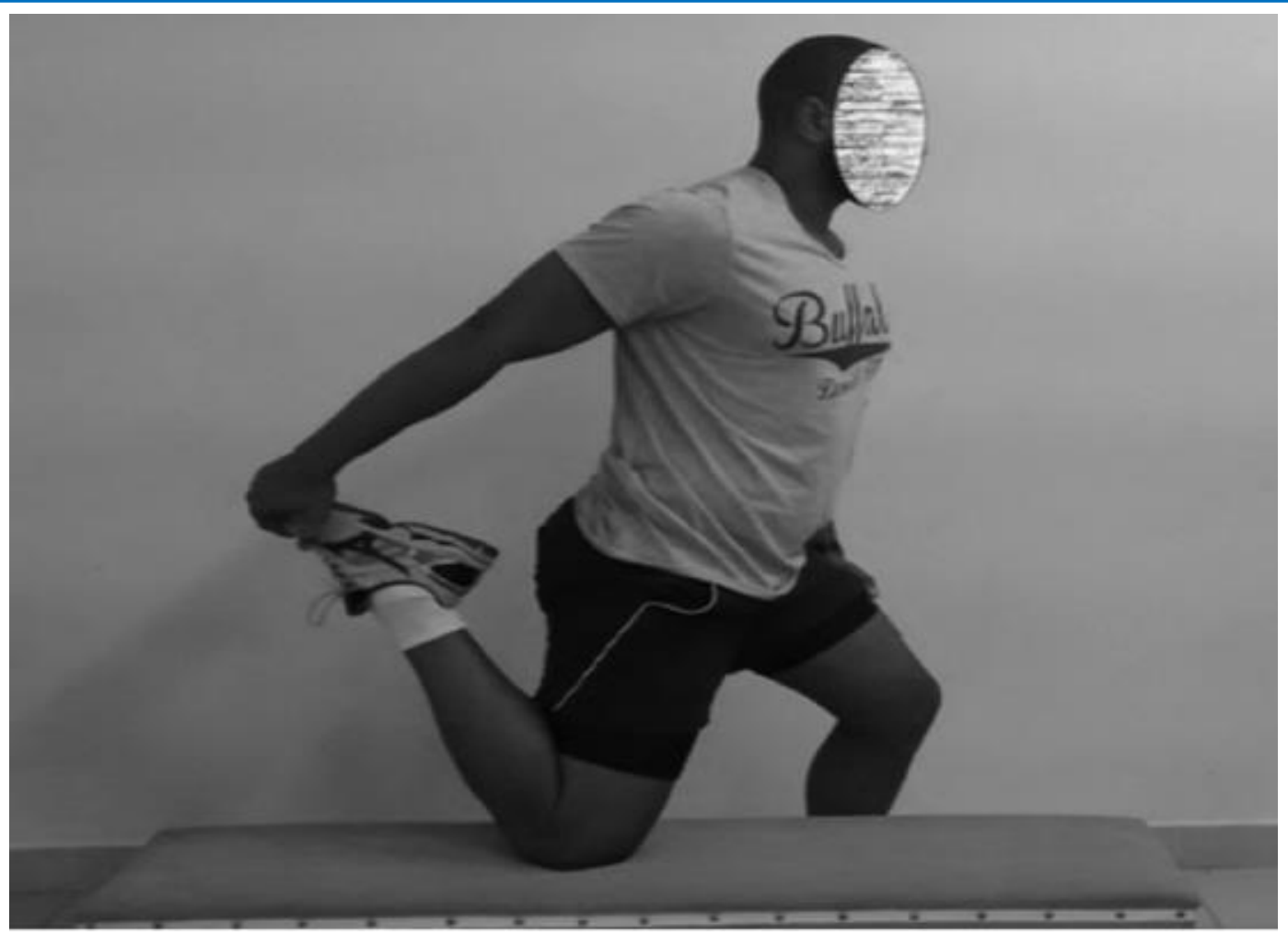

Fig 2. Stretching maneuver 


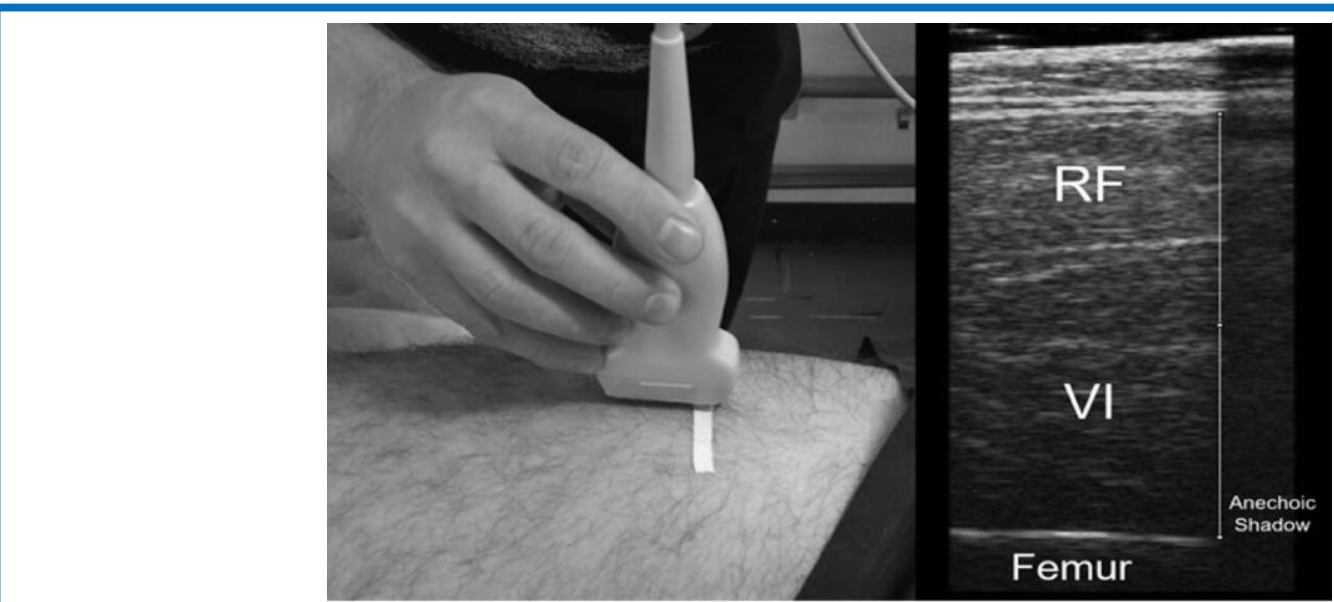

Fig. 3 Ultrasound imaging for muscle swelling measurement. A thin strip of tape was used as reference, creating an anechoic shadow allowing the repeated measures to be done at the same spot. RF: rectus femoris, VI: vastus intermedius.

extensions of each protocol, participants rested for three minutes for blood lactate measurement, which was followed by the muscle swelling measurement. Knee extensors peak torque (PT) and the total work were recorded during each protocol using the Biodex software (version 2.63). The PT was recorded from each set and was then used to calculate the fatigue index (FI) as follows: FI $(\%)=\left[\left(\mathrm{PT} 7^{\text {th }}\right.\right.$ set $/ \mathrm{PT} 1^{\text {st }}$ set $\left.) * 100\right]$. The total work $(\mathrm{J})$ (torque $\times$ distance) from each exercise protocol was expressed by the sum of work performed in each set.

\section{Surface Electromyography}

Superficial EMG signals were recorded from the rectus femoris muscle (Miotool 400 MIOTEC, Biomedical Equipaments Ltd.). Following the SENIAM recommendations, bipolar electrodes (30 $\mathrm{mm}$ apart) were positioned at $50 \%$ of the distance between anterior superior iliac spine and the superior aspect of the patella with the reference electrode positioned on the $\mathrm{C} 7$ vertebra. The skin surface was shaved, slightly abraded, and cleaned with alcohol swabs before electrode placement. The positioning site was marked with indelible ink to assure consistent electrode placement throughout all exercise sessions. Data were sampled at $2,000 \mathrm{~Hz}$ with a gain of 2,000. The signals were filtered by a $4^{\text {th }}$ order, zero-lag Butterworth filter with a bandpass of 20 to $500 \mathrm{~Hz}$. Analyses were performed in 15millisecond windows, extracted from the middle part of each concentric muscle action. The amplitude and frequency of each EMG window were described using the root mean square and median frequency, respectively. Signal amplitude was normalized by EMG amplitude acquired from a maximum voluntary isometric contraction (MVIC) performed before each isokinetic test. The MVIC was determined at $60^{\circ}$ of knee flexion and the participants had two attempts of four seconds to reach the maximum torque with one minute of rest between attempts. All signal analyses were performed in a customized Matlab program (version 6.5).

\section{Muscle Swelling Assessment}

Muscle thickness was measured via B-Mode ultrasound (Philips-VMI, Ultra Vision Flip, model BF), as an indicator of acute muscle swelling. ${ }^{12,22-24} \mathrm{~A}$ water-soluble transmission gel was applied on the $10-\mathrm{MHz}$ ultrasound probe to provide acoustic contact without depressing the dermal surface. The probe was positioned longitudinally in the midline of the anterior thigh at $50 \%$ of the distance from anterior superior iliac spine to the superior aspect of the patella. The site measured was marked with a permanent marker and tape at baseline to keep the same probe positioning after exercise and for the following exercise sessions (Figure 3). For baseline measurements, participants were evaluated in the supine position after 5min of resting. Ultrasound images were stored and further analyzed using Image-J software (National Institute of Health, USA, version 1.49). Muscle swelling was determined as the distance between the subcutaneous adipose tissue-rectus femoris interface and vastus intermedius-bone interface. All measurements were performed three times by the same investigator and the mean value was used for further analyses.

\section{Blood Lactate}

To measure blood lactate response to exercise, $25 \mu \mathrm{L}$ samples were collected from the ear lobe in capillary tubes before and 3-min after each exercise. The ear lobe was cleaned with alcohol and wiped with a sterile swab. All blood samples were deposited in Eppendorf tubes containing $50 \mu \mathrm{L}$ of sodium fluoride $(1 \%)$ and were stored at $-20{ }^{\circ} \mathrm{C}$ until further analyses. The blood lactate was later quantified using a YSI lactate analyzer (Model YSI 2300, Yellow Springs Instrument, OH, USA). 
Table 1. Electromyographic activity from rectus femoris muscle during isokinetic exercise protocols. Data are presented as mean \pm standard deviation for each of the seven sets.

\begin{tabular}{|c|c|c|c|c|c|c|c|}
\hline & \multicolumn{7}{|c|}{ Amplitude (\%MVIC) } \\
\hline Protocols & $1^{\text {st }}$ & $2^{\text {nd }}$ & $3^{\text {rd }}$ & $4^{\text {th }}$ & $5^{\text {th }}$ & $6^{\text {th }}$ & $7^{\text {th }}$ \\
\hline ISS & $135.1 \pm 20.6$ & $129.4 \pm 17.2$ & $126.4 \pm 25.3$ & $123.1 \pm 25.2$ & $121.9 \pm 24.3$ & $120.3 \pm 25.9$ & $121.7 \pm 24.5$ \\
\hline CON & $128.8 \pm 20.0$ & $128.5 \pm 24.5$ & $129.1 \pm 25.2$ & $125.7 \pm 23.8$ & $127.7 \pm 33.2$ & $128.1 \pm 31.5$ & $127.1 \pm 30.2$ \\
\hline \multirow[t]{3}{*}{ TRA } & $137.3 \pm 26.7$ & $139.6 \pm 27.3$ & $132.9 \pm 32.1$ & $138.1 \pm 29.2$ & $136.8 \pm 35.6$ & $138.5 \pm 34.4 *$ & $136.6 \pm 27.3^{*}$ \\
\hline & \multicolumn{7}{|c|}{ Median Frequency $(\mathrm{Hz})$} \\
\hline & $1^{\text {st }}$ & $2^{\text {nd }}$ & $3^{\text {rd }}$ & $4^{\text {th }}$ & $5^{\text {th }}$ & $6^{\text {th }}$ & $7^{\text {th }}$ \\
\hline ISS & $74.7 \pm 12.0$ & $69.2 \pm 11.0$ & $65.6 \pm 12.8$ & $64.1 \pm 12.6$ & $61.9 \pm 12.8$ & $61.9 \pm 11.5$ & $62.8 \pm 13.3$ \\
\hline CON & $75.8 \pm 15.2$ & $73.4 \pm 13.3$ & $70.8 \pm 12.2 *$ & $69.0 \pm 11.9 *$ & $67.7 \pm 12.5^{*}$ & $66.7 \pm 13.1$ & $66.5 \pm 12.5$ \\
\hline TRA & $74.9 \pm 14.3$ & $75.8 \pm 13.1$ & $73.9 \pm 12.3 *$ & $73.0 \pm 10.3 *$ & $72.0 \pm 10.8 *$ & $72.9 \pm 10.3 *$ & $73.2 \pm 10.5 *$ \\
\hline
\end{tabular}

ISS = Inter-set agonist static stretching; CON = short passive rest interval; TRA = Traditional passive long rest interval; RMS = Root Mean Square; \% MVIC = percent of electromyography amplitude acquired from a maximum voluntary isometric contraction; *Greater than ISS.

\section{Statistical Analyses}

Data are presented as mean \pm standard deviation. The Shapiro-Wilk test was applied to test data distribution. The amount of total work and the FI from each exercise protocol were compared using a one-way repeated measure analysis of variance (ANOVA). EMG activity, muscle thickness, and blood lactate were analyzed with a two-way repeated measure ANOVA (exercise protocols $\times$ sets). A Bonferroni adjustment was used as post hoc test if any interaction was found. All analyses were performed in SPSS (version 17.0) and an alpha level of 0.05 was adopted.

\section{Results}

For the total work, there was a main effect for exercise protocol $\left(\mathrm{F}=15.868 ; p<0.001 ; \eta_{p}{ }^{2}=0.59\right.$; Figure $\left.3 \mathrm{a}\right)$. The total work was lesser $(p=0.001)$ in ISS $(11823 \pm$ $1735 \mathrm{~J})$ compared with CON $(13976 \pm 2378 \mathrm{~J})$ and TRA $(15511 \pm 2251 \mathrm{~J})$. No difference between CON and TRA was found $(p=0.20)$.
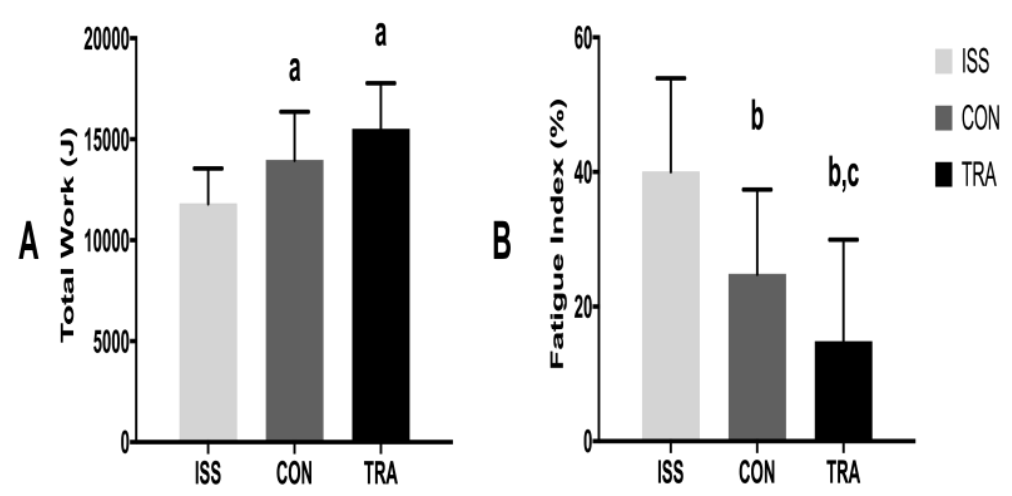

Fig. 4 Total Work and Fatigue Index (mean \pm SD). ISS: Inter-set agonist static stretching; CON: short passive rest interval; TRA: long passive rest interval (a) Greater than ISS; (b) Lesser than ISS; (c) Lesser than CON ( $p<0.05)$. 

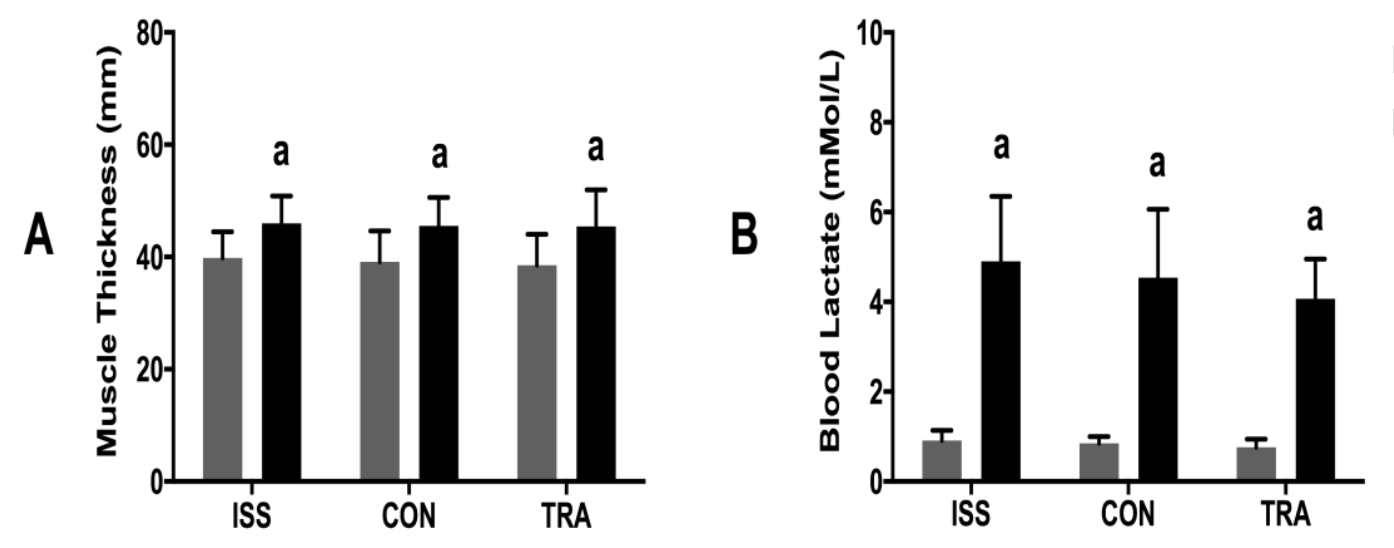

Fig. 5 Muscle Thickness and Blood Lactate (mean \pm SD); ISS: Inter-set agonist static stretching; CON: short passive rest interval; TRA: long passive rest interval (a) Greater than pre-exercise ( $p<0.05)$.

For FI, there was a main effect for exercise protocol $(\mathrm{F}=$ 16.557; $p<0.001 ; \eta_{p}{ }^{2}=0.60$; Figure 3b). The FI was greater $(p<0.05)$ in ISS $(40 \pm 14 \%)$ than CON $(25 \pm$ $13 \%)$ and TRA $(15 \pm 15 \%)$. Also, FI was greater in CON than TRA $(p=0.01)$.

There was no main effect for exercise protocol $(\mathrm{F}=$ $\left.2.430 ; p=0.11 ; \eta_{p}{ }^{2}=0.18\right)$ or set $(\mathrm{F}=0.785 ; p=0.59$; $\left.\eta_{p}{ }^{2}=0.07\right)$ for EMG amplitude. However, there was an exercise protocol $\times$ set interaction $(\mathrm{F}=1.926 ; p=0.04$; $\left.\eta_{p}{ }^{2}=0.15\right)$, where EMG amplitude was lower during the $6^{\text {th }}$ and $7^{\text {th }}$ sets during the ISS than TRA $(p<0.05$, table 1). For EMG frequency, there was a main effect for exercise protocol $\left(\mathrm{F}=7.605 ; p=0.003 ; \eta_{p}{ }^{2}=0.41\right)$ and for set $\left(\mathrm{F}=13.303 ; p<0.001 ; \eta_{p}{ }^{2}=0.55\right)$. There was also an exercise protocol $\times$ set interaction $(\mathrm{F}=5.114 ; p$ $\left.<0.001 ; \eta_{p}{ }^{2}=0.32\right)$. EMG frequency from the $3^{\text {rd }}$ to $5^{\text {th }}$ set compared were lower in ISS than CON $(p<0.05)$ and from the $3^{\text {rd }}$ to $7^{\text {th }}$ set compared to TRA $(p<0.05)$. No differences between sets were found between CON and TRA for EMG frequency $(p>0.05)$.

The pre-exercise muscle thickness was $39.83 \pm 4.64 \mathrm{~mm}$, $39.10 \pm 5.53 \mathrm{~mm}, 38.52 \pm 5.54 \mathrm{~mm}$ to ISS, CON, and TRA protocols, respectively. In addition, the postexercise muscle thickness was $45.97 \pm 4.87 \mathrm{~mm}, 45.52 \pm$ $5.05 \mathrm{~mm}, 45.44 \pm 6.49 \mathrm{~mm}$ to ISS, CON, and TRA protocols, respectively. There was no difference between pre-test muscle thickness values $(\mathrm{p}>0.05)$. However, there was a main effect for time $(\mathrm{F}=124.053 ; p<0.001$; $\eta_{p}{ }^{2}=0.92$, figure $\left.4 \mathrm{~A}\right)$. The exercise protocols showed a similar increase in muscle thickness after the exercise sessions $(p<0.001)$. Since there was neither a significant exercise protocol $\times$ time interaction $(\mathrm{F}=0.380 ; \mathrm{p}=0.69$; $\eta \mathrm{p} 2=0.03)$ nor an effect for exercise protocol $(\mathrm{F}=0.944$; $\mathrm{p}=0.40 ; \eta \mathrm{p} 2=0.08)$ in muscle thickness..

The pre-exercise blood lactate was $0.91 \pm 0.23 \mathrm{mMol} / \mathrm{L}$, $0.85 \pm 0.15 \mathrm{mMol} / \mathrm{L}, 0.75 \pm 0.18 \mathrm{mMol} / \mathrm{L}$ to ISS, CON, and TRA protocols, respectively. In addition, the post- exercise blood lactate was $4.90 \pm 1.45 \mathrm{mMol} . \mathrm{L}, 4.54 \pm$ $1.52 \mathrm{mMol} / \mathrm{L}, 4.07 \pm 0.88 \mathrm{mMol} / \mathrm{L}$ to ISS, CON, and TRA protocols, respectively. There was neither a significant exercise protocol $\times$ time interaction $(\mathrm{F}=$ 2.166; $\left.p=0.14 ; \eta_{p}{ }^{2}=0.17\right)$ nor an effect for exercise protocol $\left(\mathrm{F}=3.232 ; p=0.06 ; \eta_{p}{ }^{2}=0.23\right)$. However, there was a main effect for time $\left(\mathrm{F}=122.514 ; p<0.001 ; \eta_{p}{ }^{2}\right.$ $=0.92$, figure $4 \mathrm{~B}$ ) in blood lactate. Therefore, the protocols induced a similar increase $(p<0.001)$ in blood lactate after the exercise sessions.

\section{Discussion}

Increase in time under muscle tension during resistance strength training is thought important in maximizing the hypertrophic response of the skeletal muscle. Thus, according to some authors, implementing ISS may enhance the hypertrophic effect by increasing total time under muscle tension and associated neuromechanical, and metabolic stimuli. ${ }^{4,5}$ However, the main findings of the present study indicated that ISS increase muscle fatigue, reduce muscle activation, and force generation, as reported by lower EMG mean frequency, total work, and greater FI than TRA and CON protocols, accompanied by lower EMG amplitude in ISS than TRA. Moreover, no differences were evident in muscle swelling or blood lactate between the protocols. Together, these data indicate that ISS likely decrease the amount of force generation during subsequent sets, decreasing work output. At the same time, ISS did not promote greater metabolite accumulation and muscle swelling when compared to the CON and TRA. Therefore, it appears that ISS is not better than passive rest intervals when aiming to increase the metabolic response to ST. Based on previous theoretical approach and in vivo studies, a stretch would increase metabolite response and muscle swelling., ${ }^{5,14,25}$ It could be speculated that ISS would increase the time in which the agonist's muscles remain under tension. ${ }^{4,5}$ Furthermore, 
passive stretching has been reported to reduce blood flow through the stretched muscle because of elongation of the vessels running parallel to the muscle fibers and an increase in intramuscular pressure. ${ }^{26}$ Thus, the stretching maneuver may have disrupted regional blood flow, and therefore reduce oxygen delivery and metabolites removal. Hypothetically, it would results in a metabolite accumulation and fluid shift into the cell, increasing swelling. 5,13,14,25 However, our results indicated that metabolites responses and muscle swelling were the same between ISS, CON and TRA protocols. Thus, the reduction in blood flow is probably not the only factor that contributes to muscle swelling and a metabolite response in ST protocols. Work volume is another aspect which influences metabolite and swelling adaptations. ${ }^{27}$ In fact, Jenkins et al. ${ }^{10}$ reported a higher muscle swelling in ST protocol with greater total work. Therefore, our data suggest that a possible influence in metabolic response and muscle swelling induced by the theoretical increase in time under tension and blood flow reductions in ISS protocol might be compensated by greater work volume (mechanical stimuli) achieved during passive rest intervals ST protocols. The greater FI and lower total work observed in ISS than TRA could be partially attributed to shorter rest interval length since shorter rest intervals limit phosphorylcreatine resynthesis 28 and decrease skeletal muscle force capabilities. However, stretching of the agonist muscles during the rest intervals also decreased ST performance - as indicated by a greater FI and lower total work in ISS compared to CON protocol. This decrease in muscular performance corroborates with previous studies showing some degree of acute neuromuscular impairment following static stretching. ${ }^{20,29,30}$ Supporting the above-mentioned evidence that ISS increase muscle fatigue in ST, in the present study ISS induce less EMG amplitude in the $6^{\text {th }}$ and $7^{\text {th }}$ sets compared to TRA. Additionally, ISS attenuated EMG median frequency to a greater extent than passive rest intervals. EMG median frequency, from the $3^{\text {rd }}$ to $7^{\text {th }}$ set, was $11 \%$ to $15 \%$ lower in ISS than TRA. Since EMG median frequency, from $3^{\text {rd }}$ to $5^{\text {th }}$ sets, was also $7 \%$ to $8 \%$ lower in ISS than CON, we could speculate that static stretching was indeed responsible for this decrease in EMG median frequency. Therefore, considering that EMG amplitude and EMG median frequency provide information regarding motor unit recruitment, and firing frequency, ${ }^{10,15}$ that influence force generation, ${ }^{9}$ our data indicate that stretching applied on agonist muscles during rest interval in ST induce muscle fatigue. Previous studies have been already reported that stretching maneuver reduces neuromuscular excitation and performance. ${ }^{17,30,31}$ Trajano et al. ${ }^{17}$ reported a decrease in EMG median frequency in sustained submaximal isometric contraction after stretching. Moreover, Trajano et al. ${ }^{30}$ demonstrated a decrease in EMG amplitude and torque in evoked contraction after five minutes of static stretching. Although these studies do not resemble static stretching applied during rest interval in ST, both studies, reinforces that stretch reduces muscle force generation. In this sense, the decrease in neuromuscular performance likely occurred due to neural aspects. ${ }^{32-36}$ In this regard, Trajano et al. ${ }^{29}$ observed that the central factors of the muscle's control play the most substantial role in force loss induced by stretching exercise. Additionally, static stretching can decrease muscle spindle sensitivity and motor neuron excitability due to overstimulation of type III and IV joint receptors. ${ }^{37}$ In such scenarios, both passive tension and myotendinous stiffness might be reduced, ${ }^{38}$ impairing the force transfer from muscle to bone, resulting in the observed lower torque output. The muscle hypertrophy is one of the most pursued adaptations by ST practitioners. In this sense, the increase of metabolite byproduct accumulation and muscle swelling, which describe the metabolite stress caused by ST, play an important role in muscle hypertrophy. ${ }^{8}$ In addition, motor unit recruitment and firing frequency, which raise force generation and total work increasing mechanotransduction, are essential to muscle growth. ${ }^{9}$ Therefore, the knowledge concerning the effects of ISS in metabolic stress and mechanotransduction might help explain the chronic adaptations on muscle hypertrophy and strength gains. Although, the chronic response to ISS remains without conclusive evidence, ${ }^{7}$ our data suggest that ISS might not be the best strategy for muscle hypertrophy. Since ISS reduces mechanotransduction without amplifying metabolic responses.

In conclusion, the ISS protocol induced similar metabolite accumulation and muscle swelling with a decrease in muscle activation, force production, and work output.

\section{List of acronyms}

CON - control rest interval (subject passively rested between sets for $40 \mathrm{sec}$ )

EMG - electromyography

FI - fatigue index

ISS - intra-set static stretching

$\mathrm{J}$ - joules

MVC - maximal voluntary contraction

MVIC - maximum voluntary isometric contraction

PT - peak torque

ST - strength-training

TRA - traditional passive rest interval (subject passively rested between sets for $120 \mathrm{sec}$ )

\section{Authors contributions}

UCP participated in the conception and design of the work, data acquisition and analysis, drafting of the manuscript, and approved the final version. AV participated in the conception and design of the work, data analysis, drafting of the manuscript, and approved the final version. DCLV participated in the data analysis, drafting of the manuscript, and approved final version. FDdL participated in the data analysis, drafting of the manuscript, and approved the final version. VARJr 
participated in the data analysis, drafting of the manuscript, and approved the final version. JJT participated in the drafting of the manuscript, revised of the English grammar, and approved the final version. MB participated in the conception and design of the work, data analysis, drafting of the manuscript, and approved the final version.

\section{Acknowledgments None.}

Funding No funding was obtained for this project.

\section{Conflict of Interest}

The authors have no conflicts to disclose.

\section{Ethical Publication Statement}

We confirm that we have read the Journal's position on issues involved in ethical publication and affirm that this report is consistent with those guidelines.

\section{Corresponding Author}

Denis César Leite Vieira

Faculdade de Educação Física, Universidade de Brasília, Campus Universitário Darcy Ribeiro, Asa Norte, Brasilia, DF, Brasil. Zipcode: 70910-970.

Phone: + 55 (61) 3107-2522;

Email: denisclvieira@hotmail.com

\section{E-mails of co-authors}

Ubiratan Contreira Padilha: ucpadilha@outlook.com Amilton Vieira: acmribeirao@gmail.com Filipe Dinato De Lima: fdinatolima@gmail.com Valdinar Araújo Rocha Jr:valdinar@gmail.com James J Tufano: james.j.tufano@gmail.com Martim Bottaro: martim.bottaro@gmail.com

\section{References}

1. Mazzeo RS, Cavanagh P, Evans WJ, et al. ACSM Position Stand: Exercise and Physical Activity for Older Adults. Med Sci Sport Exerc 1998;30:9921008.

2. Fleck SJ, Kraemer WJ. Designing Resistance Training Programs. 4th ed. Champaign-Urbana: Human Kinects, 2014.

3. Angleri V, Ugrinowitsch C, Libardi CA. Crescent pyramid and drop-set systems do not promote greater strength gains, muscle hypertrophy, and changes on muscle architecture compared with traditional resistance training in well-trained men. Eur J Appl Physiol 2017;117:359-69.

4. Souza AC, Bentes CM, De Salles BF, et al. Influence of inter-set stretching on strength, flexibility and hormonal adaptations. J Hum Kinet 2013;36:127-35.

5. Mohamad NI, Nosaka K, Cronin J. Maximizing hypertrophy: Possible contribution of stretching in the interset rest period. Strength Cond J 2011;33:81-7.

6. Beardsley C. How do advanced techniques affect hypertrophy? https://medium.com/@SandCResear ch/how-do-advanced-techniques-affect-hypertroph y-8fed29705d13 (2019).

7. Evangelista AL, De Souza EO, Moreira DCB, et al. Interset Stretching vs. Traditional Strength Training. J Strength Cond Res 2019;26:1.

8. Schoenfeld BJ. Potential mechanisms for a role of metabolic stress in hypertrophic adaptations to resistance training. Sport Med 2013;43:179-94.

9. Schoenfeld BJ. The mechanisms of muscle hypertrophy and their application to resistance training. J Strength Cond Res 2010;24:2857-72.

10. Jenkins NDM, Housh TJ, Bergstrom HC, et al. Muscle activation during three sets to failure at 80 vs. $30 \%$ 1RM resistance exercise. Eur J Appl Physiol 2015;115:2335-47.

11. Sjogaard G, Adams RP, Saltin B. Water and ion shifts in skeletal muscle of humans with intense dynamic knee extension. Am J Physiol Integr Comp Physiol 1985;248:R190-6.

12. Vieira A, Blazevich A, Souza N, et al. Acute changes in muscle thickness and pennation angle in response to work-matched concentric and eccentric isokinetic exercise. Appl Physiol Nutr Metab 2018;43:1069-74.

13. Freitas EDS, Poole C, Miller RM, et al. Time Course Change in Muscle Swelling: High-Intensity vsBlood Flow Restriction Exercise. Int J Sports Med 2017;38:1009-16.

14. Poole DC, Musch TI, Kindig CA. In vivo microvascular structural and functional consequences of muscle length changes. Am J Physiol - Hear Circ Physiol 1997;272:H2107-14.

15. Dankel SJ, Mattocks KT, Jessee MB, et al. Do metabolites that are produced during resistance exercise enhance muscle hypertrophy? Eur J Appl Physiol 2017;117:2125-35.

16. Fowles JR, Sale DG, MacDougall JD. Reduced strength after passive stretch of the human plantarflexors. J Appl Physiol 2000;89:1179-88.

17. Trajano G, Pinho C, Costa P, et al. Static stretching increases muscle fatigue during submaximal sustained isometric contractions. J Sports Med Phys Fitness 2015;55:43-50.

18. Kay AD, Blazevich AJ. Concentric muscle contractions before static stretching minimize, but do not remove, stretch-induced force deficits. J Appl Physiol 2010;108:637-45.

19. Kay AD, Blazevich AJ. Effect of acute static stretch on maximal muscle performance: A systematic review. Med Sci Sports Exerc 2012;44:154-64.

20. Behm DG, Chaouachi A. A review of the acute effects of static and dynamic stretching on performance. Eur J Appl Physiol 2011;111:263351.

21. Barroso R, Tricole V, Gil S dos S, et al. Maximal Strength, number of repetitions, and total volume are differently affected by static-,ballistic-, and proprioceptive neuromuscular facilitation stretching. J Strength Cond Res 2012;26:2432-7. 
22. Yasuda T, Fukumura K, Iida H, et al. Effect of lowload resistance exercise with and without blood flow restriction to volitional fatigue on muscle swelling. Eur J Appl Physiol 2015;115:919-26.

23. Jessee MB, Mattocks KT, Buckner SL, et al. The acute muscular response to blood flow-restricted exercise with very low relative pressure. Clin Physiol Funct Imaging 2018;38:304-11.

24. Vieira A, Siqueira AF, Ferreira-Junior JB, et al. Ultrasound imaging in women's arm flexor muscles: Intra-rater reliability of muscle thickness and echo intensity. Brazilian $\mathbf{J}$ Phys Ther 2016;20:535-42.

25. McDaniel J, Ives SJ, Richardson RS. Human muscle length-dependent changes in blood flow. J Appl Physiol 2012;112:560-5.

26. Otsuki A, Fujita E, Ikegawa S, et al. Muscle oxygenation and fascicle length during passive muscle stretching in ballet-trained subjects. Int $\mathrm{J}$ Sports Med 2011;32:496-502.

27. Figueiredo VC, de Salles BF, Trajano GS. Volume for Muscle Hypertrophy and Health Outcomes: The Most Effective Variable in Resistance Training. Sport Med 2017;48:1-7.

28. Harris RC, Edwards RHT, Hultman E, et al. The time course of phophorylcreatine resynthesis during recovery of the quadriceps muscle in man. Pflugers Arch 1976;367:137-42.

29. Trajano GS, Seitz L, Nosaka K, et al. Contribution of central vs. peripheral factors to the force loss induced by passive stretch of the human plantar flexors. J Appl Physiol 2013;115:212-8.

30. Trajano GS, Seitz LB, Nosaka K, et al. Can passive stretch inhibit motoneuron facilitation in the human plantar flexors? J Appl Physiol 2014;117:1486-92.

31. Sekir U, Arabaci R, Akova B, et al. Acute effects of static and dynamic stretching on leg flexor and extensor isokinetic strength in elite women athletes. Scand J Med Sci Sport 2010;20:268-81.

32. Márquez G, Romero-Arenas S, Marín-Pagán C, et al. Peripheral and central fatigue after high intensity resistance circuit training. Muscle Nerve 2017;56:152-9.

33. Junior RM, Berton R, de Souza TMF, et al. Effect of the flexibility training performed immediately before resistance training on muscle hypertrophy, maximum strength and flexibility. Eur J Appl Physiol 2017;117:767-74.

34. Marek SM, Cramer JT, Fincher AL, et al. Acute effects of static and proprioceptive neuromuscular facilitation stretching on muscle strength and power output. J Athl Train 2005;40:94-103.

35. Miranda H, Maia MDF, Paz GA, et al. Acute effects of antagonist static stretching in the inter-set rest period on repetition performance and muscle activation. Res Sport Med 2015;23:37-50.

36. Opplert J, Babault N. Acute Effects of Dynamic Stretching on Muscle Flexibility and Performance: An Analysis of the Current Literature. Sport Med 2017;48:1-27.

37. Avela J, Kyröläinen H, Komi P V. Altered reflex sensitivity after repeated and prolonged passive muscle stretching. J Appl Physiol 1999;86:128391.

38. Kubo K, Kanehisa H, Kawakami Y, et al. Influence of static stretching on viscoelastic properties of human tendon structures in vivo. J Appl Physiol 2001;90:520-7.

Submission: September 26, 2019

Revision received: October,11, 2019

Acceptance: October 19, 2019 